\title{
Salvage definitive chemo-radiotherapy for locally recurrent oesophageal carcinoma after primary surgery: Retrospective review
}

\author{
SH Baxi, ${ }^{1}$ B Burmeister, ${ }^{1}$ JA Harvey, ${ }^{1}$ M Smithers ${ }^{2}$ and J Thomas ${ }^{2}$ \\ ${ }^{1}$ Department of Radiation Oncology, Princess Alexandra Hospital and University of Queensland, and ${ }^{2}$ Department of Surgery, \\ 2 Princess Alexandra Hospital, Brisbane, Queensland, Australia
}

\section{SUMMARY}

To determine the overall survival and gastrointestinal toxicity for patients treated with salvage definitive chemoradiotherapy after primary surgery for locoregional relapse of oesophageal carcinoma. A retrospective review of 525 patients who had a resection for oesophageal or oesophagogastric carcinoma at Princess Alexandra Hospital identified 14 patients treated with salvage definitive radiotherapy or chemo-radiotherapy, following localized recurrence of their disease. We analysed the patient and treatment characteristics to determine the median overall survival as the primary end point. Gastrointestinal toxicity was examined to determine if increased toxicity occurred when the stomach was irradiated within the intrathoracic radiotherapy field. The median overall survival for patients treated with curative intent using salvage definitive chemo-radiotherapy was 16 months and the 2-year overall survival is $21 \%$. One patient is in clinical remission more than 5 years after therapy. Age $<60$ years old and nodal recurrence were favourable prognostic factors. Treatment compliance was $93 \%$ with only one patient unable to complete the intended schedule. Fourteen per cent of patients experienced grade 3 or 4 gastrointestinal toxicity. Salvage definitive chemo-radiotherapy should be considered for good performance status patients with oesophageal carcinoma who have a locoregional relapse after primary surgery. The schedule is tolerable with low toxicity and an acceptable median survival.

Key words: recurrent oesophageal carcinoma; salvage chemo-radiotherapy.

\section{INTRODUCTION}

Carcinoma of the oesophagus is a rare disease in most countries although its incidence in the developed world is rising rapidly. ${ }^{1}$ This is mainly because of an increase in adenocarcinoma, which typically arises in the distal oesophagus or gastro-oesophageal junction. The optimal management in terms of local control is surgical resection although local recurrence and the development of distant metastases remain a problem. For patients unable to have surgery, radical chemo-radiotherapy is an alternative, although its efficacy is most marked in squamous cell carcinomas and in the prox- imal two thirds of the oesophagus. ${ }^{2}$ For tumours that are resectable, a recent meta-analysis clearly shows an improvement in outcomes with the use of both neoadjuvant chemotherapy and neoadjuvant chemo-radiotherapy. ${ }^{2}$ Despite these efforts to improve results with neoadjuvant therapy, there are a proportion of patients who fail locoregionally without evidence of systemic metastasis. In these patients, there is a potential for cure, accepting that the risk of further distant relapse remains high.

For oesophageal tumours, the issue of salvage therapy in the event of locoregional recurrence is rarely considered. Head

SH Baxi MB BS; B Burmeister FRANZCR; JA Harvey FRANZCR; M Smithers FRACS; J Thomas BHSc.

Correspondence: Dr Siddhartha H Baxi, Department of Radiation Oncology, Princess Alexandra Hospital, 199 Ipswich Road, Woolloongabba, QLD 4102, Australia. Email: siddhartha_baxi@ hotmail.com

4 Conflicts of interest: None.

3 Submitted XX XX XXXX; accepted XX XX XXXX.

doi: 10.1111/j.1440-1673.2008.02023.X

(C) 2008 The Authors

Journal compilation (c) The Royal Australian and New Zealand College of Radiologists

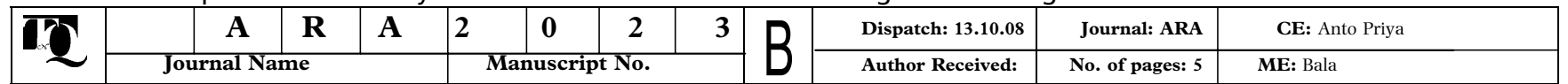


and neck cancer represents an example of where locoregional failures can possibly be salvaged by radiotherapy after initial surgery. ${ }^{3}$ For the oesophagus, one of the obstacles to delivering curative radiotherapy is whether the patient has been treated with neoadjuvant radiotherapy and what dose has been delivered to radiosensitive tissues such as the spinal cord. In addition, the delivery of very high doses of radiation to the oesophagus and trachea may result in strictures. Furthermore, the question of whether high-dose radiotherapy in this setting results in greater gastric toxicity as the stomach is positioned within the irradiated field in the thorax needs to be addressed, especially if the recurrence is at the anastomosis. At this time, there are no published data relating to this problem.

From a large series of patients who have had various treatment modalities of surgery, radiotherapy and chemotherapy for oesophageal cancer, we aimed to assess the outcomes of patients who have had high-dose salvage chemo-radiotherapy after having relapsed locoregionally in the chest or lower neck after initial resection of their tumour.

\section{METHODS}

A database of all patients with cancer of the oesophagus or oesophagogastric junction has been maintained since 1987 through the Princess Alexandra Hospital Combined Upper Gastrointestinal Oncology Clinic. Patient demographics, investigations, management, pathology and disease status are prospectively documented from the time of presentation. Following definitive treatment, patients are routinely followed up, with investigations carried out when clinically indicated by patient's symptoms and signs.

\section{Eligibility}

From the database, we identified the patients who had a documented locoregional recurrence following initial curative surgery for an oesophageal cancer. Patients may have had neoadjuvant therapy. Those patients who subsequently received salvage definitive chemo-radiotherapy with curative intent were reviewed. To receive salvage chemo-radiotherapy, patients had to be Eastern Cooperative Oncology Group performance status 0 or 1 , have locoregional disease and be able to receive a reference dose of more than $50 \mathrm{~Gy}$. Locoregional recurrence was defined as anastomotic recurrence or mediastinal lymphadenopathy.

Eligible patients were reviewed in terms of initial pathology, initial management, patterns of relapse, type of salvage therapy, consequent toxicity and outcome. Particular detail was given to documenting neoadjuvant therapy, surgical technique and morbidity resulting from the salvage therapy. Staging involved the use of endoscopy, transluminal endoscopic ultrasound (when available) and CT scanning. Positron emission tomography was used when it became available to exclude distant metastases.

\section{Salvage treatment schedules}

\section{Radiation technique}

Patients were planned using conformal three-dimensional CT planning and treated supine in a shell using a three- or fourfield, two-phase technique with lung inhomogeneity correction. The gross tumour volume was covered with a margin of 2$3 \mathrm{~cm}$. Total doses varied between 45 and 60 Gy over 25-30 fractions (5-6 weeks) at 1.8-2.2 Gy per fraction. The biological effective dose for all schedules was calculated to be more than 50 Gy. Spinal cord tolerance was kept to $<48$ Gy with lung tolerances of $V_{20}<30 \%$.

\section{Chemotherapy schedules}

When used, the chemotherapy schedule involved 5-fluorouracil (5FU) with or without cisplatin and depended on co-morbidities. The schedules included $225 \mathrm{mg} / \mathrm{m}^{2} /$ day $5 \mathrm{FU}$ continuous infusion concurrent with radiotherapy or $800 \mathrm{mg} / \mathrm{m}^{2} /$ day $5 \mathrm{FU}$ for 4 days and $80 \mathrm{mg} / \mathrm{m}^{2}$ cisplatin on weeks 1 and 5 of radiotherapy or $80 \mathrm{mg} / \mathrm{m}^{2}$ cisplatin only on weeks 1 and 5 of radiotherapy.

\section{End-points}

Survival was determined from the date of initial diagnosis and from the diagnosis of the locoregional recurrence to death. Recurrence was defined as local if anastomotic or regional if involving the mediastinal lymph nodes (including the supraclavicular lymph nodes as long as they were treated within the radiation field to a curative dose). The initial position of the tumour was defined according to upper, middle and lower thirds of the thoracic oesophagus. Survival was analysed by the Kaplan-Meier method. Statistical analysis was carried out using the Statistical Package for Social Science 14.1(SPSS, Chicago, IL). We carried out a univariate analysis to determine whether age, gender, histologic subtype, initial site, overall stage, previous neoadjuvant therapy, site of relapse or type of salvage therapy predicted for any adverse outcome. Continuous data were compared by the Mann-Whitney $U$ test and the ordinal data by the $\chi^{2}$ test. The study complied with the Australian National Health and Medical Research Council's guidelines on research involving human subjects. Toxicity was assessed using the CTCAE version 3.0.

\section{RESULTS}

Of the 1065 patients treated between 1987 and 2005, 525 $(47.9 \%)$ had primary resection with or without neoadjuvant radiotherapy, $238(21.7 \%)$ had definitive radiotherapy or chemo-radiotherapy and $302(26.7 \%)$ received treatment with palliative intent. Neoadjuvant treatment involved a radiation dose of 35 Gy in 15 fractions over 3 weeks with concurrent $5 \mathrm{FU}$ and cisplatin in week 1 of radiotherapy. From the patients who had a resection, $46(9.1 \%)$ had a documented locoregional relapse. From this group, 14 (2.7\%) were considered suitable to 
receive definitive salvage radiotherapy or chemo-radiotherapy. The other 32 were treated with palliative intent using chemotherapy, radiotherapy, stenting or supportive care only. This was primarily because of the use of prior radiotherapy, performance status and co-morbidities. The 14 patients who had curative salvage therapy had a mean follow-up time of 13 months (3-118 months). The majority were male (79\%) over 60 years of age. Fifty per cent had stage 3 disease at first presentation, with predominantly adenocarcinomas (71\%) located in the mid-oesophagus. Relapses after initial surgery were mostly in the mediastinal lymph nodes (50\%). These details are summarized in Table 1. Neoadjuvant therapy was used in $50 \%$ of the patients with one having a complete response. All patients had resection of the oesophagus with the mediastinal, subcarinal, left gastric and celiac nodes. Most salvage schedules involved combined chemo-radiotherapy (79\%), with a median dose of $60 \mathrm{~Gy}$ in 30 fractions. Concurrent cisplatin and $5 \mathrm{FU}$ were used in $36 \%$ of patients, while a single agent was used in $36 \%$. One patient had excision of a supraclavicular lymph node followed by regional radiotherapy. These details are summarized in Table 2.

Of the three patients who received neoadjuvant chemoradiotherapy initially, cumulative spinal cord doses after salvage radiotherapy were available for two of them. These two patients received 48.5 and 50 Gy to the spinal cord, which indicated two minor departmental protocol violations. The possible toxicity was accepted by the treating team and patient to achieve the required dose to the target volume.

The median overall survival for the group from the time of detection of the recurrence was 16 (3-172) months with one patient surviving more than 5 years after salvage therapy. The

6 Table 1. Patient characteristics $(N=14)$

\begin{tabular}{lc}
\hline Characteristic & $n(\%)$ \\
\hline Age in years, median (range) & $62.9(46-70)$ \\
Male & $11(79)$ \\
Histology & \\
$\quad$ Adenocarcinoma & $10(71)$ \\
Squamous cell carcinoma & $4(29)$ \\
Initial site & \\
Upper third & $4(29)$ \\
Middle third & $7(50)$ \\
Lower third & $3(21)$ \\
Stage following surgery & \\
pCR & $1(7)$ \\
1 & $3(21)$ \\
2A & $2(15)$ \\
$2 B$ & $1(7)$ \\
3 & $7(50)$ \\
Site of relapse & \\
Anastomotic & $4(29)$ \\
Mediastinal lymph nodes & $7(50)$ \\
Supraclavicular lymph nodes & $3(21)$ \\
\hline
\end{tabular}

Journal compilation (c) The Royal Australian and New Zealand College of Radiologists
Table 2. Prior and current therapy

\begin{tabular}{lc}
\hline Therapy & $n(\%)$ \\
\hline Prior neoadjuvant therapy & $7(50)$ \\
None & $4(29)$ \\
Chemotherapy alone & $3(21)$ \\
Chemo-radiotherapy & \\
Salvage therapy & $11(79)$ \\
$\quad$ Chemo-radiotherapy & $2(14)$ \\
Radiotherapy & $1(7)$ \\
Radiotherapy and surgery & $11(79)$ \\
Salvage radiotherapy & $3(21)$ \\
58-60 Gy in 28-30 fractions & \\
Others† & $4(29)$ \\
Salvage chemotherapy & $5(36)$ \\
Continuous fluorouracil & $1(7)$ \\
Cisplatin and fluorouracil & $3(21)$ \\
Cisplatin alone & \\
No chemotherapy &
\end{tabular}

†45 Gy in 20 fractions, 50.4 Gy in 28 fractions and 50 Gy in 25 fractions.

median overall survival from diagnosis of the primary oesophageal cancer was 36 (10-86) months. These results are shown in Figures 1 and 2. On univariate analysis, only age ( $>60$ years) and site of recurrence (anastomotic recurrence) predicted for a poorer outcome. These results are shown in Table 3.

Toxicity of salvage therapy was generally acceptable. The most notable side-effects were related to oesophagitis and gastritis with five patients experiencing this toxicity (one with grade 3 oesophagitis). These results are summarized in Table 4. There were no radiotherapy-related treatment breaks, with all 14 patients completing the course as intended. There were no toxicity-related hospital admissions or cases of pneumonitis in this cohort.

\section{DISCUSSION}

Data on the survival benefit of salvage chemo-radiotherapy for locoregional recurrent oesophageal cancer after initial surgery

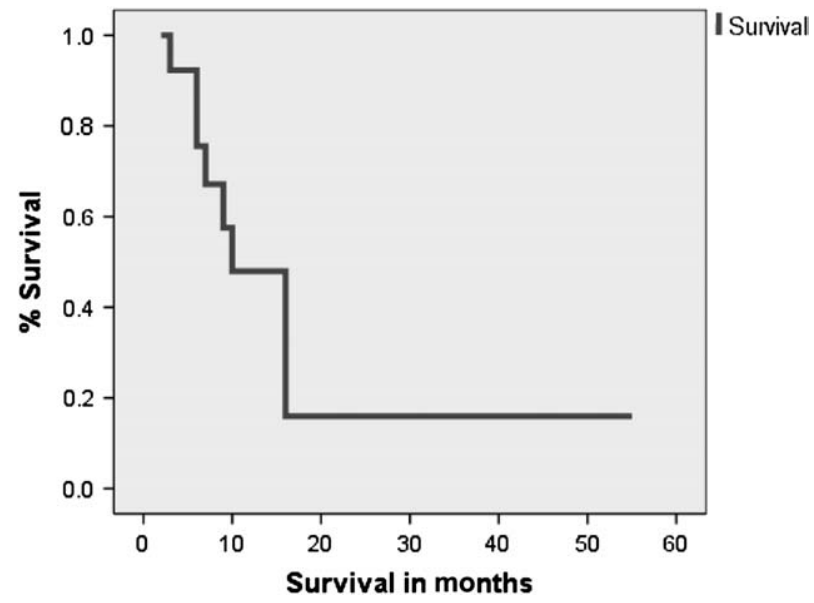

Fig. 1. Survival from date of recurrence to death. 


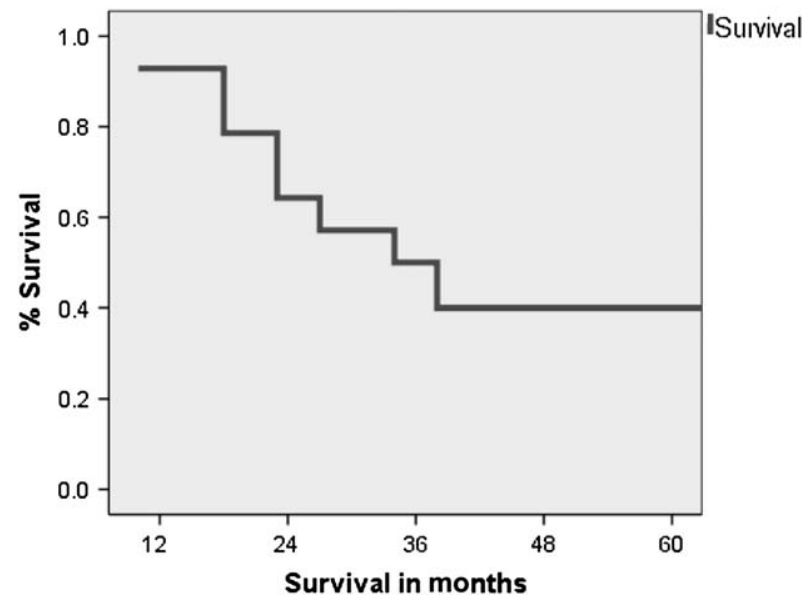

Fig. 2. Survival from date of diagnosis to death.

are scarce. We report a $9 \%$ locoregional recurrence rate after definitive surgery with one third of those (or $2.6 \%$ of those who had a primary resection) being suitable for salvage definitive chemo-radiotherapy with curative intent. This is clearly a highly selected group of patients who were considered fit and able to tolerate combination therapy with a potential for cure. Most recurrences do not occur in a setting where they are salvageable by radical curative doses of radiotherapy or chemo-radiotherapy. For those who are fit and agreeable to undergo such treatment, salvage radiotherapy offers a small but real chance of inducing a second long-term remission.

There have been other reported studies involving salvage radiation therapy for patients in this setting, usually with concurrent chemo-radiotherapy. The majority of these reports are from Japan, involving patients with squamous cell carcinomas. $^{4-7}$ There are reports of a 2 -year survival of $19 \%$ with a median survival of 9.5 and 13.8 months, respectively. ${ }^{5,6}$ The study by Nishimura et al. looked at the feasibility and effectiveness of concurrent chemo-radiotherapy for postoperative locally recurrent oesophageal cancer-analysing 16 patients using a dose of 60 Gy in 30 fractions over 7 weeks with $5 \mathrm{FU}$

Table 3. Univariate analysis of prognostic variables for overall survival

\begin{tabular}{ll}
\hline Variable & $P$-value \\
\hline Age: $<60$ vs $>60$ years & 0.019 \\
Gender: Male vs female & 0.496 \\
Histologic subtype: adenocarcinoma vs squamous & 0.712 \\
$\quad$ cell carcinoma & \\
Initial site: upper vs middle vs lower & 0.08 \\
Overall stage & 0.07 \\
Neoadjuvant therapy: none vs chemotherapy vs & 0.8 \\
$\quad$ chemo-radiotherapy & \\
Site of relapse: anastomosis vs mediastinal nodes & 0.03 \\
Salvage therapy received: chemo-radiotherapy vs & 0.579 \\
$\quad$ radiotherapy & \\
\hline
\end{tabular}

Table 4. Acute gastric toxicity

\begin{tabular}{ll}
\hline Toxicity & Number \\
\hline Nausea & $3(1 \mathrm{G} 3)$ \\
Vomiting & 0 \\
Anorexia & 2 \\
Oesophagitis/gastritis & $5(1 \mathrm{G} 3)$ \\
\hline
\end{tabular}

(250-300 $\mathrm{mg} / \mathrm{m}^{2} /$ day on weeks $1,3,5$ and 7) and fractionated cisplatin (10 mg/day over 5 days on weeks 1,2, 5 and 6 ). The compliance rate was $72 \%$ with a response rate of $72 \%$. This regimen proved to be well tolerated and provided some extension of survival compared with palliative measures with no significant grade 3 or 4 non-haematological toxicity.

In 2006, Jingu et al. prospectively assessed 30 patients treated with salvage chemo-radiotherapy following previous resection. They reported a median survival of 39 months and a 3-year survival of $56.3 \%{ }^{4}$ Their regimen used nedaplatina cisplatin-based derivative which has shown efficacy in phase II studies. These figures have yet to be confirmed in other studies. Our data are the first series to include patients with adenocarcinomas of the oesophagus. This may explain the inferior median survival when compared with that series.

Despite the fact that the numbers are small in all the reports, the predominance of adenocarcinomas in our series may have affected relapse patterns and survival. Nevertheless in this highly selected group where restaging after locoregional recurrence shows localized disease, an aggressive approach to management seems justified. We found that age and the site of recurrence were significant predictors of survival and traditional predictors of outcome such as histologic subtype and type of salvage therapy warrant further evaluation in larger studies. These prognostic variables were similar to those reported by Jingu et al. who found that performance status, age and pattern of recurrences influenced prognosis. Similar to our finding, it appears that mediastinal recurrences do better than anastomotic recurrences. Tumour subtype, location, stage and the use of neoadjuvant chemo- or chemo-radiotherapy did not have a significant impact on survival. None of the patients in this series had two synchronous localized sites of recurrence although it must be emphasized that this was not a selection criterion. Certainly, patients who have two localized sites of disease encompassable by single radiation portal are also candidates for radical salvage treatment. However, as this is a small and selected series, it is difficult to define the clinical significance of the identified variables on a univariate analysis. Whether these adverse prognostic factors should be considered for more intensive treatment is unclear. We can only postulate that perhaps, anastomotic recurrences do worse than nodal recurrences because of the influence on the ability to eat and maintain adequate nutrition and a stable weight. Ideally, a prospective randomized controlled trial would be required to Journal compilation (c) The Royal Australian and New Zealand College of Radiologists 
8 Table 5. Comparative salvage chemo-radiotherapy studies

\begin{tabular}{|c|c|c|c|c|}
\hline Study authors & Study type & No. patients & Tumour subtypes & Median OS (months) \\
\hline Yamashita et al. ${ }^{6}$ & Retrospective & 16 & SCC & 13.8 \\
\hline Nishimura et al. ${ }^{5}$ & Retrospective & 13 & SCC & 9.5 \\
\hline Jingu et al. ${ }^{4}$ & Prospective & 30 & SCC & 39 \\
\hline Nemoto et al. ${ }^{7}$ & Retrospective & 33 & SCC & 7 \\
\hline Present study & Retrospective & 14 & Mixed & 16 \\
\hline
\end{tabular}

further evaluate the survival benefits identified in this retrospective series and further clarify the prognostic significance of the variables discussed above. However, as presented, this is a small subset of patients and such a trial is probably not feasible.

There was no inference from these data that gastric toxicity is increased with the presence of the stomach in the irradiated field within the thorax. There was a 15\% (2 of 14) rate of grade 3 or 4 gastrointestinal toxicity presenting as nausea and heartburn. It is possible that a relative decrease in blood supply to the transposed stomach may result in less radiation damage and therefore explain these findings. In addition, conformal radiation fields may also contribute to the low toxicity observed. Despite having had previous therapy, we felt that the outcome in these patients was surprisingly good and that the principle of salvage radical chemo-radiotherapy is applicable to oesophageal cancer as it is in other forms of cancer.

\section{CONCLUSION}

We conclude that the median overall survival of 16 months for definitive salvage radiotherapy with or without chemotherapy is tolerable and that this approach is justifiable in this small group of highly selected patients. Age and site of recurrence appear to influence outcomes with statistical significance. There is no trend towards greater toxicity with the stomach being within the irradiated field within the thorax.

\section{ACKNOWLEDGEMENTS}

We acknowledge all the clinicians, nurses and allied health staff not mentioned as authors for their support in conducting this study.

\section{REFERENCES}

1. Bollschweiler E, Wolfgarten E, Gutschow $C$ et al. Demographic variations in the rising incidence of oesophageal adenocarcinoma in white males. Cancer 2001; 92: 549-55.

2. Gebski V, Burmeister $\mathrm{B}$, Smithers $\mathrm{M}$ et al. Surgery alone versus chemo-radiotherapy followed by surgery for resectable cancer of the oesophagus. Lancet Oncol 2006; 6: 659-68.

3. Stevens K, Britsch A, Moss WT. High-dose reirradiation of head and neck cancer with curative intent. Int J Radiat Oncol Biol Phys 1994; 29: 687-98.

4. Jingu $\mathrm{K}, \mathrm{Nemoto} \mathrm{K}$, Matsushita $\mathrm{H}$ et al. Results of radiation therapy combined with nedaplatin and 5-FU for post-operative loco-regional recurrent oesophageal cancer. BMC Cancer 2006; 6: 501-9.

5. Nishimura $\mathrm{Y}$, Koike $\mathrm{T}$, Nakamatsu $\mathrm{K}$ et al. Concurrent chemoradiotherapy with protracted infusion of 5-FU and cisplatin for post-operative recurrent or residual oesophageal cancer. Jpn J Clin Oncol 2003; 33: 341-5.

6. Yamashita $\mathrm{H}$, Nakagawa $\mathrm{M}$, Nakamura $\mathrm{N}$ et al. Salvage radiotherapy for postoperative loco-regional recurrence of oesophageal cancer. Dis Oesophagus 2005; 18 215-20.

7. Nemoto K, Hisanori A, Yoshihisa K et al. Radiation therapy for locoregionally recurrent esophageal cancer after surgery. Radiother Oncol 2001; 61: 165-8. 


\section{Author Query Form}

\section{Journal: Journal of Medical Imaging and Radiation Oncology}

\section{Article : ara 2023}

Dear Author,

During the copy-editing of your paper, the following queries arose. Please respond to these by marking up your proofs with the necessary changes/additions. Please write your answers on the query sheet if there is insufficient space on the page proofs. Please write clearly and follow the conventions shown on the attached corrections sheet. If returning the proof by fax do not write too close to the paper's edge. Please remember that illegible mark-ups may delay publication.

Many thanks for your assistance.

No.

1 Author: Please check the short title inserted (not more than 40 characters including spaces).

2 Author: Please check the edits made to the affiliations.

3 Wiley-Blackwell: Please provide the accepted dates.

$4 \quad$ Author: Please provide details of potential conflicts of interest, if any.

$5 \quad$ Author: Please check the Reference 4 page range.

6 Author: Please define "pCR" in the footnote of Table 1.

$7 \quad$ Author: Please note that Table 5 is not cited in the text. Please check.

8 Author: Please define 'OS' and 'SCC' in the footnote of Table 5. 